\title{
Effects of Body Pillow Use on Sleeping Posture and Sleep Architecture in Healthy Young Adults
}

\author{
Insung Park, PhD ${ }^{1}$, Chihiro Suzuki, MMSc ${ }^{1}$, Yoko Suzuki, PhD, RPSGT' ${ }^{1}$, Fusae Kawana, BSc, RPSGT ${ }^{1,2}$, \\ Katsuhiko Yajima, PhD³ , Shoji Fukusumi, PhD¹, Toshio Kokubo, PhD11, Kumpei Tokuyama, PhD1, \\ Masashi Yanagisawa, MD, $\mathrm{PhD}^{1}$, Makoto Satoh, MD, $\mathrm{PhD}^{1}$ \\ ${ }^{1}$ International Institute for Integrative Sleep Medicine (WPI-IIIS), University of Tsukuba, Ibaraki, Japan \\ ${ }^{2}$ Cardiovascular Respiratory Sleep Medicine, Juntendo University Graduate School of Medicine, Tokyo, Japan \\ ${ }^{3}$ Faculty of Pharmaceutical Sciences, Josai University, Saitama, Japan
}

\author{
Received: February 8, 2021 \\ Revised: March 19, 2021 \\ Accepted: March 24, 2021 \\ Correspondence \\ Makoto Satoh, $\mathrm{MD}, \mathrm{PhD}$ \\ International Institute for Integrative Sleep \\ Medicine (WPI-IIIS), \\ University of Tsukuba, 1-2 Kasuga, Tsukuba, \\ Ibaraki 305-8550, Japan \\ Tel +81-29-859-1858 \\ Fax +81-29-859-1290 \\ E-mail satoh.makoto.fu@u.tsukuba.ac.jp \\ ORCID \\ Insung Park \\ https://orcid.org/0000-0001-6389-1937 \\ Chihiro Suzuki \\ https://orcid.org/0000-0002-7272-0299 \\ Yoko Suzuki \\ https://orcid.org/0000-0001-7957-1743 \\ Fusae Kawana \\ https://orcid.org/0000-0003-2227-0549 \\ Katsuhiko Yajima \\ https://orcid.org/0000-0002-8199-9981 \\ Shoji Fukusumi \\ https://orcid.org/0000-0002-0641-9799 \\ Toshio Kokubo \\ https://orcid.org/0000-0002-9098-3690 \\ Kumpei Tokuyama \\ https://orcid.org/0000-0003-1756-4227 \\ Masashi Yanagisawa \\ https://orcid.org/0000-0002-7358-4022 \\ Makoto Satoh \\ https://orcid.org/0000-0002-2723-8153
}

(c) This is an Open Access article distributed under the terms of the Creative Commons Attribution Non-Commercial License (https://creativecommons.org/licenses/by-nc/4.0) which permits unrestricted non-commercial use, distribution, and reproduction in any medium, provided the original work is properly cited.
Background and Objective Patients with position-dependent obstructive sleep apnea have a $>2$-fold higher apnea-hypopnea index when sleeping in a supine position compared with a nonsupine position. We investigated the effect of body pillow use on sleeping body position and sleep architecture in healthy young adults.

Methods In experiment 1, we evaluated the body pressure distribution with or without body pillow use in 8 healthy young adults [age, $36.5 \pm 13.0$ years; body mass index (BMI); $20.6 \pm 1.2 \mathrm{~kg} /$ $\mathrm{m}^{2}$ ]. In experiment 2 , we performed a randomized-crossover intervention study to evaluate the effects of body pillow use on sleeping position and sleep architecture in 10 healthy young adults (age, $24.3 \pm 7.8$ years; BMI, $21.4 \pm 1.7 \mathrm{~kg} / \mathrm{m}^{2}$ ). Sleep architecture was characterized by polysomnography, and body positions were monitored using a sensor. Subjective sleep quality was evaluated with the Oguri-Shirakawa-Azumi sleep inventory, middle age and aged version.

Results In experiment 1, body pillow use significantly reduced mean body pressure on the shoulder, hip, and whole body. In experiment 2 , mean time spent in the supine, lateral, and prone body positions did not differ significantly between the 2 trials. Body pillow use, however, significantly extended the sustained time spent in the lateral body position compared with the control trial. Subjective sleep quality and sleep architecture did not differ significantly between the 2 trials, but body pillow use decreased the number of short (30 s) slow-wave sleep episodes.

Conclusions Sleeping with a body pillow effectively extends sustained time in a lateral sleeping position and prevents segmentation of slow-wave sleep episodes.

Sleep Med Res 2021;12(1):57-63

Key Words Body pillow, Sleeping body position, Lateral position, Sleep architecture, Energy expenditure.

\section{INTRODUCTION}

Obstructive sleep apnea (OSA) is a common sleep-linked breathing disorder characterized by recurrent episodes of upper airway disruption during sleep [1]. Effective treatment of OSA reduces medical risk factors associated with cardiovascular and cerebrovascular disease, and metabolic syndrome [2,3]. Current guidelines recommend continuous positive airway pressure (CPAP) as the gold standard for managing the clinical state of OSA [4]. CPAP improves sleep quality, subjective or/and objective daytime sleepiness, and mood, especially in those with severe OSA [5]. The use of CPAP decreases complaints of excessive daytime sleepiness and functional impairment due to hypersomnolence [6,7]. In patients with OSA, CPAP decreases the sleep latency, the percent time in non-rapid eye movement (NREM) stage 1, the arousal index, 
and the respiratory disturbance index, and increases the percent time in NREM stage 2, thereby also affecting the sleep architecture [8,9]. Although CPAP is as an effective treatment for upper airway obstruction in sleep apnea, the treatment is not always tolerated and compliance with CPAP therapy is limited; $29 \%$ to $83 \%$ of patients are nonadherent [10]. The main reasons for noncompliance with CPAP management are the side effects, which include dry nose and mouth, sneezing, nasal congestion, skin irritation, abrasions on the nose bridge, and eye irritation from mask leaks [11].

Patients with position-dependent OSA (POSA) have a greater than 2-fold higher apnea-hypopnea index (AHI) during sleep in the supine position compared with a non-supine position [12]. Patients with POSA are estimated to comprise $70 \%$ to $80 \%$ of those with mild (AHI $\left.=5-14 \mathrm{hr}^{-1}\right)$ and moderate OSA (AHI = $\left.15-29 \mathrm{hr}^{-1}\right)$, and approximately $56 \%$ to $75 \%$ of patients with severe OSA (AHI $\geq 30 \mathrm{hr}^{-1}$ ) [13-15]. In POSA, sleeping in the supine position is associated with worse OSA [16], and many forms of positional therapy have been developed in an attempt to improve long-term adherence, e.g., chest-worn sleep position trainers, tennis ball technique, positional alarms, and verbal instructions urging patients to shift to a non-supine body position when the supine body position is detected [17-19]. These treatments induce pain and discomfort, and subsequently disrupt the sleep architecture, which also limit compliance. As an alternative method, a specially designed body pillow was developed to enforce the lateral position, but its efficacy has not been evaluated [20].
The most likely reason for disrupting a lateral sleeping position is excess body pressure distribution on the shoulder and hip [21].

We hypothesized that using a body pillow improves body pressure distribution, which extends the duration of a sustained lateral sleeping body position. In the present study, we evaluated the effect of body pillow use on sleeping body position, sleep architecture, and sleeping energy metabolism in healthy young adults.

\section{METHODS}

\section{Protocol and Subjects}

For experiment 1, we recruited 8 healthy young adults [mean \pm standard deviation (SD) age $36.5 \pm 13.0$ years, mean body mass index (BMI) $20.6 \pm 1.2 \mathrm{~kg} / \mathrm{m}^{2}$ ] to evaluate the distribution of body pressure in the supine, prone, and lateral sleeping positions. The temporary body pressure distribution was measured in each sleep position using an ERGOCHECK system (ABW Co. Ltd., Hamburg, Germany) in all participants. The measurements were obtained with the participants awake and under the investigator's specific instructions. An example of the body pressure distribution in each position is shown in Fig. 1.

Experiment 2 was a randomized-crossover intervention study with or without body pillow use, which were separated a washout period of more than 1 week. One week preceding the experiment, the participants stayed in a whole room metabolic chamber
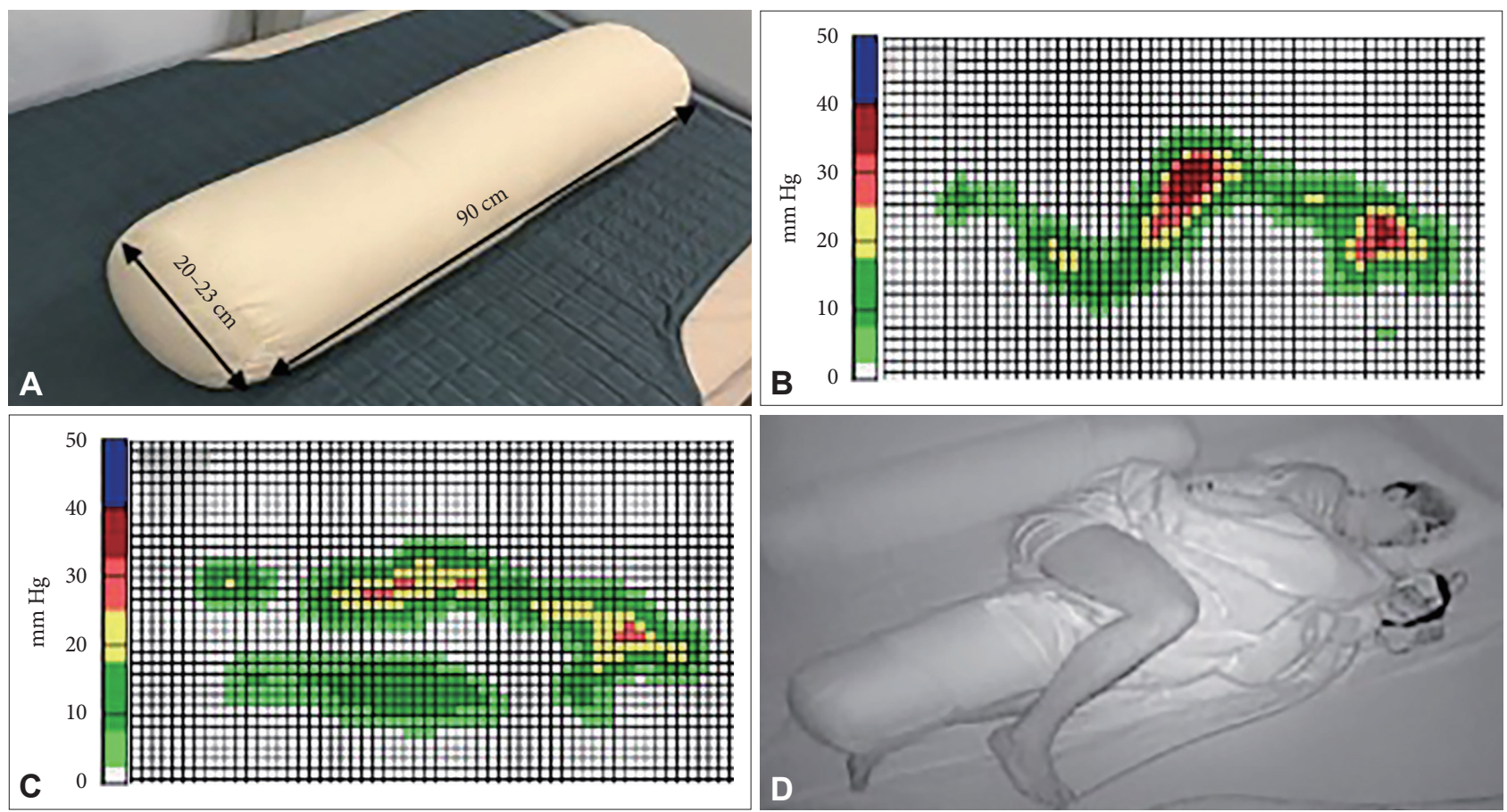

Fig. 1. Body pillow, body pressure distribution, and example of body pillow use during sleep. A: Body pillow used in this study. Participants were provided the opportunity to choose the appropriate body pillow for their body volume among the S-, M-, and L-sized body pillows. B, C: Examples of body pressure distribution without and with body pillow use. Each grid represents $5 \times 5 \mathrm{~cm}$. D: Example of body pillow use during sleep in experiment 2 . Note that 2 pillows were used, 1 on each side of the body. 
as an adaptation night, during which the sensors and electrodes of the polysomnographic (PSG) recording system were attached to the participants. Three days prior to the experiment, the participants were instructed to maintain a constant $8 \mathrm{~h}$ sleep/16 h wake schedule following their habitual bed and awake time and to refrain from taking naps, which was confirmed by wrist actigraphy (ActiGraph, Ambulatory Monitoring, NY, USA), and to complete daily diaries. Participants refrained from ingesting caffeine and alcohol for 1 week prior to the experiment. All participants, 10 healthy young adults (4 male, 6 female), satisfied the inclusion criteria: 20 to 65 years of age, BMI 18.5 to 25 (kg/ $\mathrm{m}^{2}$ ), a regular sleep/wake pattern, and not engaged in regular exercise more than twice a week. Exclusion criteria were as follows: self-reported sleep problems (Pittsburgh Sleep Quality Index score $>5$ ); shiftwork or transmeridian travel within 3 months before the study; smoking; excessive alcohol intake (> 30 g/day); currently taking medication for cardiovascular disease, hypertension, diabetes, hypercholesterolemia, hyperglycemia, or hyperlipidemia; use of medications affecting sleep or metabolism; and severe sleep apnea syndrome. The Morningness-Eveningness Questionnaire (MEQ) was administered to eliminate participants having an extreme morning and evening type (41 < MEQ scores < 59) [22].

On the experiment day, participants arrived at the laboratory after dinner. Electrodes for polysomnography and a body position sensor were attached before the participants entered the metabolic chamber where they remained sedentary until their habitual sleep onset time. Participants went to bed at their habitual bedtime and slept for $8 \mathrm{~h}$. Participants responded to a questionnaire about subjective sleep quality after waking the following morning.

The study was conducted according to the guidelines for procedures involving human subjects put forth by the Declaration of Helsinki, and the protocol was approved by the Ethics Committee of the University of Tsukuba (H30-284). All of the participants provided written informed consent before study initiation.

\section{Body Pillow}

The body pillow was designed to support the upper side of the arm and leg to reduce body pressure on the lower side of shoulder and hip (Fig. 1). The body pillows (Nishikawa Co., Ltd., Tokyo, Japan) were made from $100 \%$ polyester. All participants were provided the opportunity to choose from among 3 body pillow sizes according to their body volume (S: diameter $20 \mathrm{~cm}$, length $90 \mathrm{~cm}$, weight $1.19 \mathrm{~kg}$; M: diameter $23 \mathrm{~cm}$, length $90 \mathrm{~cm}$, weight $1.4 \mathrm{~kg}$; and L: diameter $26 \mathrm{~cm}$, length $90 \mathrm{~cm}$, weight $2.0 \mathrm{~kg}$ ). The subjects used 2 body pillows, with 1 positioned on each side of the body (Fig. 1).

\section{Polysomnography}

The recording system (PSG-1100; Nihon Kohden, Tokyo, Japan) comprised 6 electroencephalography derivations (F3-M2,
F4-M1, C3-M2, C4-M1, O1-M2, and O2-M1), submental and leg electromyography, bilateral electrooculogram, air flow at the nose and mouth, chest and abdominal respiratory movement, oxygen saturation at the fingertip, and a body position sensor that was fixed on the skin at the center of the sternum. Respiratory events such as the AHI and the oxygen desaturation index (ODI) were scored according to the American Academy of Sleep Medicine (AASM) criteria: apnea was defined as a drop in airflow of at least $90 \%$ from baseline lasting $10 \mathrm{~s}$ or longer. Hypopnea was defined as a $>30 \%$ drop in airflow lasting at least $10 \mathrm{~s}$, associated with either a decrease in arousal or $>3 \% \mathrm{O}_{2}$ saturation drop [23]. The electroencephalography electrodes were placed on the surface of the head in accordance with the international 10-20 system, recording at the frontal (F3, F4), central $(\mathrm{C} 3, \mathrm{C} 4)$, and occipital $(\mathrm{O} 1, \mathrm{O} 2)$ sites, and at 2 reference sites on M1 and M2. Sleep parameters were scored in 30-s intervals as wakefulness, and stages 1, 2, 3 [slow wave sleep (SWS)], and REM sleep according to standard criteria of the AASM [23]. In addition, total sleep time, sleep onset latency, REM sleep latency, and sleep efficiency were evaluated. The body position sensor discriminated among the 5 body positions; supine, prone, left lateral, right lateral, and sitting. During the whole sleep period, body positions were also monitored by an infrared camera.

\section{Subjective Self-Reported Quality of Sleep}

The Oguri-Shirakawa-Azumi sleep inventory, middle age and aged version (OSA-MA) questionnaire was used to assess subjective sleep quality after waking in the morning [24]. This questionnaire comprises 16 items with 5 factors ("Sleepiness on rising," "Initiation and maintenance of sleep," "Frequent dreaming," "Refreshness," and "Sleep length."

\section{Indirect Calorimetry}

The airtight metabolic chamber measures $2.00 \times 3.45 \times 2.10 \mathrm{~m}$ (FHC-15S; Fuji Medical Science Co., Ltd., Chiba, Japan), and air in the chamber is pumped out at a rate of $80 \mathrm{~L} / \mathrm{min}$. The temperature and relative humidity of incoming fresh air were controlled at $25^{\circ} \mathrm{C}$ and $55 \%$, respectively. The chamber is furnished with desk, chair, and toilet. Concentrations of oxygen $\left(\mathrm{O}_{2}\right)$ and carbon dioxide $\left(\mathrm{CO}_{2}\right)$ in the outgoing air were measured with high precision by online process mass spectrometry (VG Prima $\delta B$; Thermo Electron Co., Winsford, UK). The precision of the mass spectrometry, defined as the SD for continuous measurement of the calibrated gas mixture $\left(\mathrm{O}_{2}, 15 \% ; \mathrm{CO}_{2}, 5 \%\right)$, was $0.0016 \%$ for $\mathrm{O}_{2}$ and $0.0011 \%$ for $\mathrm{CO}_{2}$. Every minute, $\mathrm{O}_{2}$ consumption $\left(\dot{\mathrm{VO}}_{2}\right)$ and $\mathrm{CO}_{2}$ production $\left(\dot{\mathrm{VCO}}_{2}\right)$ rates are calculated using an algorithm for improved transient response [25]. Energy expenditure was calculated from $\dot{\mathrm{VO}}_{2}, \dot{\mathrm{VCO}}_{2}$, and urinary nitrogen $(\mathrm{N})$ excretion, as described previously [26]. Urinary $\mathrm{N}$ was measured using the Kjeldahl method. Energy expenditure and macronutrient oxidation were calculated from the $\dot{\mathrm{VO}_{2}}, \dot{\mathrm{V} C O}$, and urinary $\mathrm{N}$ excretion. The rate of urinary 
$\mathrm{N}$ excretion, an index of protein catabolism, was assumed to be constant during the calorimetry [27].

\section{Statistical Analysis}

Physical characteristics of the participants, sleep parameters, OSA-MA parameters, and body position are expressed as the mean (SD). Paired t-tests were used to compare the sleep parameters and OSA-MA parameters between the mean values of the trials. Time in each body position and longest duration of sustained lateral time were assessed by 1-way repeated-measures analysis of variance (ANOVA) and Bonferroni's correction for multiple comparisons. The SWS episode frequency was assessed by 2-way repeated-measures ANOVA and Bonferroni's correction for multiple comparisons. Data analysis was conducted using SPSS ver. 22 (IBM Corp., Armonk, NY, USA). A p value < 0.05 was considered statistically significant.

\section{RESULTS}

\section{Subjects Characteristics}

The mean \pm SD age, BMI, and MEQ score of the participants was $24.3 \pm 7.8$ years, $21.4 \pm 1.7 \mathrm{~kg} / \mathrm{m}^{2}$, and $51.1 \pm 7.2$, respectively. All participants fulfilled all inclusion/exclusion criteria and completed the 2 trials.

\section{Body pressure distribution}

In experiment 1 , body pillow use significantly reduced the mean body pressure on the shoulder, hip, and whole body (Table 1).

\section{Objective sleep quality}

In experiment 2 , the basic sleep architecture did not differ between the 2 trials in any of the sleep parameters (i.e., durations of stage 1,2, SWS, REM, and wakefulness after sleep onset) (Table 2). AHI and ODI were also not significantly different between the 2 experimental conditions. A 2-way ANOVA for the distribution of the SWS episode duration showed significant main effects of body pillow use $[F(1,9)=1.609, \mathrm{p}=0.24]$ and SWS episode duration $[\mathrm{F}(9,81)=35.2, \mathrm{p}<0.001]$, as well as a significant interaction of the body pillow $\times$ SWS episode duration $[\mathrm{F}(9,81)$ $=2.5, \mathrm{p}<0.05]$. Post-hoc analysis showed a significant decrease in the number of SWS episodes lasting only $30 \mathrm{~s}$ in the body pillow trial ( $<<0.001$ ) (Fig. 2). Thus, body pillow use improved deep sleep maintenance without disturbing the sleep architecture.

Table 1. Mean body pressure in each body part

\begin{tabular}{lccc}
\hline \multicolumn{1}{c}{ Parameters } & Control & Body pillow & p-value \\
\hline Shoulder $(\mathrm{mm} \mathrm{Hg})$ & $33.1 \pm 5.1$ & $28.2 \pm 4.0$ & $<0.01$ \\
Hip (mm Hg) & $41.4 \pm 8.6$ & $31.5 \pm 5.9$ & $<0.01$ \\
Whole body mean (mm Hg) & $13.0 \pm 0.7$ & $11.5 \pm 1.1$ & $<0.01$ \\
\hline
\end{tabular}

Values are mean \pm SD for control and body pillow trials.

\section{Changed sleep body position}

The mean time spent in the supine, lateral, and prone body positions did not differ significantly between the 2 trials (Fig. 3 ). The longest sustained duration of the lateral body position was significantly extended with body pillow use compared with the control trial [45.10 (7.05) vs. 70.05 (6.96) $\mathrm{min}$ for control and body pillow trials, $\mathrm{p}<0.05$ ] (Fig. 4). Thus, body pillow use did not increase the total amount of time in the lateral body position, but did prolong the sustained duration of lateral body position during sleep.

\section{Subjective sleep quality}

None of the parameters ('sleepiness on rising', 'initiation and maintenance of sleep,' 'sleep length,' 'refreshness', and 'frequent dreaming or nightmares') of subjective sleep quality assessed by the OSA-MA questionnaire differed significantly between the

Table 2. Sleep architecture

\begin{tabular}{lccc}
\hline \multicolumn{1}{c}{ Parameters } & Control & Body pillow & p-value \\
\hline Total bedtime (min) & 480.0 & 480.0 & \\
Total sleep time (min) & $442.7 \pm 41.9$ & $449.4 \pm 18.2$ & 0.45 \\
Wakefulness (min) & $31.7 \pm 41.4$ & $24.8 \pm 14.1$ & 0.51 \\
Sleep latency (min) & $5.7 \pm 3.1$ & $5.5 \pm 5.1$ & 0.92 \\
Sleep efficiency (\%) & $93.3 \pm 8.7$ & $94.8 \pm 3.0$ & 0.50 \\
Stage 1 (min) & $46.0 \pm 34.4$ & $41.8 \pm 19.0$ & 0.51 \\
Stage 2 (min) & $231.7 \pm 49.1$ & $223.9 \pm 43.4$ & 0.57 \\
SWS (min) & $89.7 \pm 33.1$ & $100.0 \pm 43.8$ & 0.26 \\
REM sleep (min) & $75.3 \pm 23.8$ & $83.8 \pm 21.6$ & 0.20 \\
REM sleep latency (min) & $23.8 \pm 15.3$ & $20.8 \pm 8.6$ & 0.11 \\
Arousal index (/night) & $168.1 \pm 90.4$ & $154.2 \pm 59.8$ & 0.29 \\
AHI (/h) & $2.3 \pm 1.7$ & $2.3 \pm 1.5$ & 0.91 \\
3\%ODI & $2.6 \pm 1.6$ & $2.2 \pm 1.6$ & 0.57
\end{tabular}

Values are mean \pm SD for control and body pillow trials. SWS: slow wave sleep, REM: rapid eye movement, AHI: apnea-hypopnea index, $3 \% \mathrm{ODI}$ : $3 \%$ oxygen desaturation index.

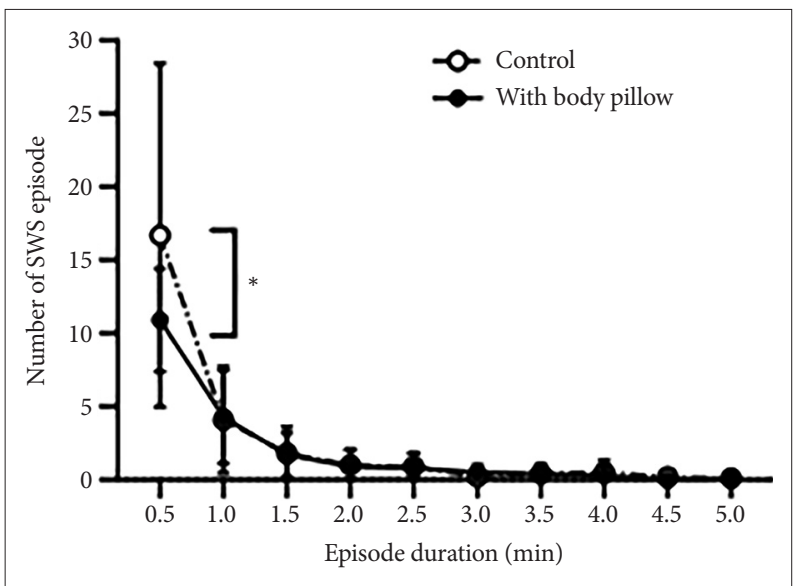

Fig. 2. Frequency of slow wave sleep (SWS) episodes lasting less than 5 min. $* p<0.05$. 


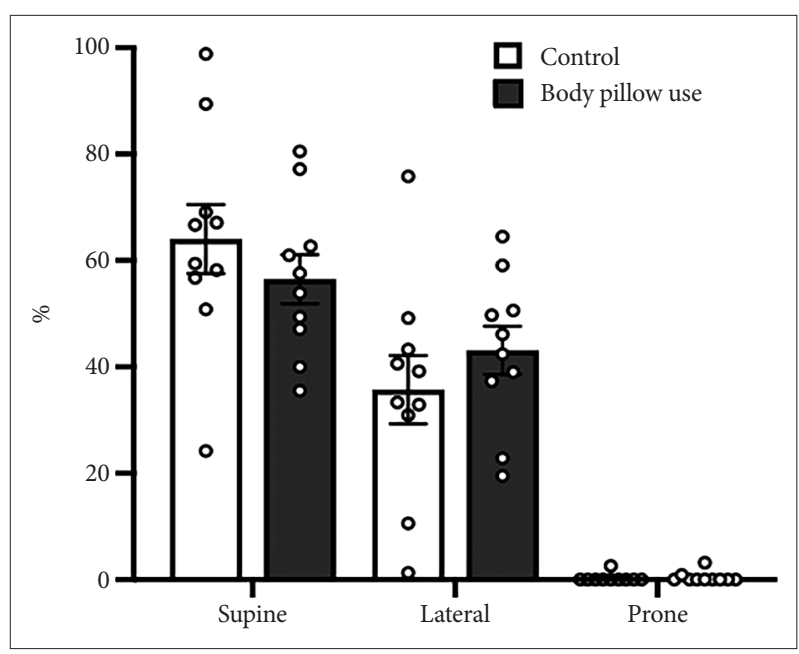

Fig. 3. Percent time in each body position between control and body pillow trials. Values are mean \pm SD for control and body pillow trials, and values from individual participants are also plotted. SD: standard deviation.

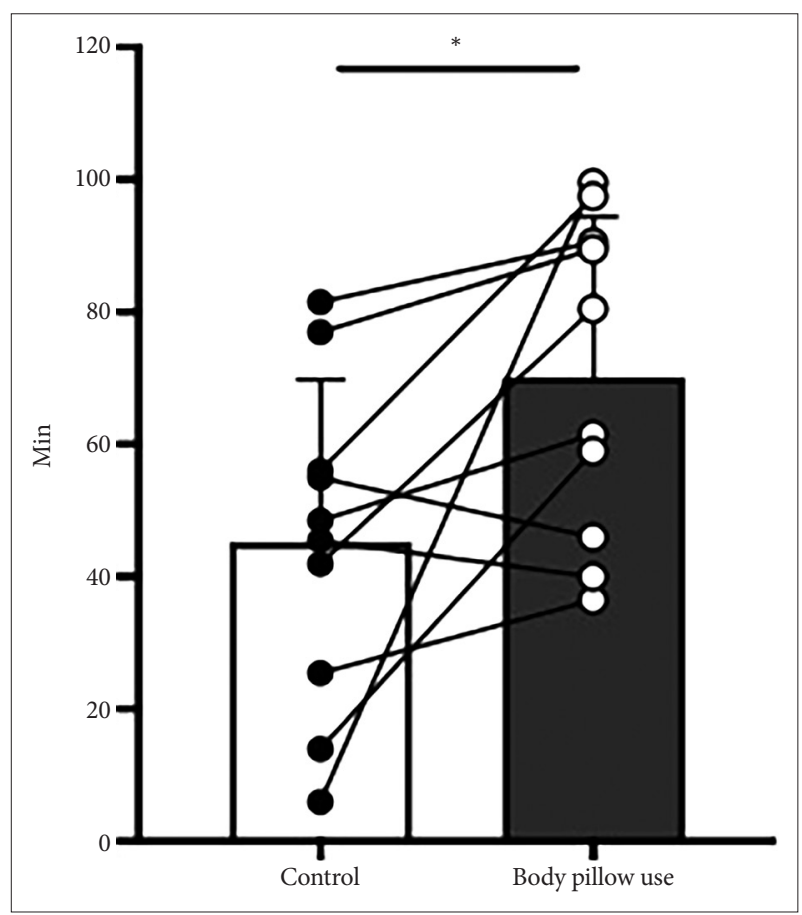

Fig. 4. Longest sustained duration of lateral body position. Values are mean \pm SD for control and body pillow trials, and values for individual participants are also plotted. ${ }^{*} p<0.05$. SD: standard deviation.

control and body pillow trials (Table 3). Therefore, the body pillow did not affect the subjective assessment of the quality of sleep.

\section{Metabolic data}

Energy expenditure [control trial: $381.99(0.17) \mathrm{kcal} / 8 \mathrm{~h}$ vs. body pillow trial: $378.81(0.17) \mathrm{kcal} / 8 \mathrm{~h}, \mathrm{p}=0.55]$ and respiratory quotient [control trial: $0.84(0.06) \mathrm{kcal} / 8 \mathrm{~h}$ vs. body pillow trial: $0.85(0.06) \mathrm{kcal} / 8 \mathrm{~h}, \mathrm{p}=0.47)$ during sleep differed between the 2 trials.
Table 3. Subjective sleep quality assessed by the OSA sleep inventory MA version

\begin{tabular}{lclc}
\hline \multicolumn{1}{c}{ Parameters } & Control & \multicolumn{2}{c}{ Body pillow } \\
\hline Sleepiness on rising & $48.7 \pm 7.7$ & $50.0 \pm 8.8$ & 0.65 \\
Sleep duration & $45.8 \pm 12.0$ & $48.3 \pm 8.2$ & 0.42 \\
$\begin{array}{l}\text { Initiation and maintenance } \\
\text { of sleep }\end{array}$ & $42.4 \pm 11.4$ & $42.8 \pm 10.3$ & 0.94 \\
Refreshness & & & \\
Frequent dreaming, nightmares & $45.2 \pm 9.1$ & $43.6 \pm 11.4$ & 0.30 \\
\hline
\end{tabular}

Values are mean \pm SD for control and body pillow trials.

OSA: The Oguri Shirakawa and Azumi standard rating scale, MA: middle age and aged version.

\section{DISCUSSION}

One of the most common and effective treatments for sleep apnea is CPAP [4]. In a previous study, the effects of positional therapy were equivalent to that of CPAP for normalizing AHI, sleep quality, and nocturnal oxygenation in patients with POSA [20]. Positional therapies are associated with side effects, however, leading to low compliance. In contrast, a mild intervention, such as body pillow use, may be free from side effects and become a habitual daily routine. The present study investigated the effect of body pillow use on sleeping body position and sleep architecture in healthy young subjects, as assessed by a sleep questionnaire, sleep staging, and body position monitoring. Our findings revealed a positive effect of body pillow use to extend the duration of a sustained lateral sleeping position and suppress fragmented SWS without interfering with the subjective sleep quality.

The frequency and severity of apnea/hypopnea events are increased in the supine body position compared with the lateral body position [28]. Therefore, maintaining a lateral body position during sleep might improve sleep apnea and the quality of natural sleep. Positional therapy using the tennis ball technique (a tennis ball is fastened to the back to discourage the patient from sleeping supine) improved subjective sleep quality and daytime alertness and decreased snoring loudness. Discomfort related to the technique, however, led to its discontinuation in $38 \%$ of the participants [28]. In another study, $80 \%$ of subjects stopped using the tennis ball technique, claiming that it was uncomfortable [18]. As an alternative approach to avoid the supine position, previous studies designed special mattresses and pillows to facilitate prone positioning, which improved the mean AHI and ODI, and decreased the time spent in the supine position of OSA patients without disruption of the sleep architecture judged by the PSG recording system $[29,30]$. The special mattresses and pillows were so effective that some subjects remained in the prone position for the entire sleeping period, i.e., no changes in sleeping position. Given the positive physiologic roles of changing the sleeping position [31-33], it is better for patients with POSA to avoid 
the supine position while not suppressing natural changes in the sleeping body position. As a less stringent intervention, use of a body pillow significantly increased the duration of sustained lateral body position while also allowing for changes in sleeping positions. The use of a body pillow improved the body pressure distribution and induced positive changes in sleeping body positions and sleep architecture without restricting changes in sleeping body positions.

In the present study, we found that body pillow use did not statistically change objective and subjective sleep parameters compared with the control trial. The absence of changes in the PSG parameters between the 2 trials might be due to the nature of the participants in this study. The participants were healthy young adults with no sleep problems such as sleep apnea. The short sleep latency, high sleep efficiency, and long SWS duration of the participants in the control trial may have resulted in a ceiling effect that masked the effect of body pillow use on sleep parameters. The number of short SWS episodes ranging from $0.5-5$ min was decreased by body pillow use, although there was no difference in the total duration of SWS between the 2 trials. Differences in the number of short SWS episodes were also observed when the effects of 2 mattress types on sleep were assessed [21]. Although, the mechanism responsible for the suppression of SWS episode fragmentation by body pillow use remains to be elucidated, analysis of the continuity of SWS episodes may provide greater insight into the quality of sleep.

In a previous study, a specially designed body pillow was used to enforce the lateral position and facilitate the prone position, which was rare in natural sleep in the present study [29,30]. The prone position during sleep increases the heart rate and respiratory quotient compared with the supine position [34] and compromises thermoregulation in infants [35]. On the other hand, it is speculated that a non-prone sleeping posture requires more energy expenditure due to the respiratory movements of the rib cage against gravity [36]. To assess the reasonable assumption that difference in the sleeping posture might induce changes in energy metabolism, indirect calorimetry was performed using a whole room metabolic chamber in the present study. Despite the high time resolution of the indirect calorimetry in the present study, there was no difference in energy expenditure between the 2 trials. According to these results, the lateral sleeping position might not only positively influence POSA, but might also suppress SWS episode fragmentation.

The present study had some limitations. First, participants were healthy young adults with no sleep problems such as sleep apnea. Thus, further studies to evaluate the efficacy of body pillow use for maintaining a desirable sleep posture should be performed in participants with OSA, particularly POSA. Second, a previous study revealed that prone positions were less likely in older persons, who demonstrate a progressive preference for lateral positions due to a decline in spinal flexibility and/or the extra effort required for breathing [37]. Third, by the nature of the interven- tion, this study design was not double-blinded, and placebo effect of pillow use on subjective sleep quality could not be excluded. These limitations will be addressed in a future study.

In conclusion, the findings of the present study demonstrate that sleeping with a body pillow effectively extends the duration of a sustained lateral sleeping position and prevents segmentation of SWS episodes.

\section{Acknowledgments}

The present study was financially supported by Nishikawa Co., Ltd. The authors would like to thank Takuto Nonomura, Yoji Shimura, and Saki Shimada of Nishikawa Co., Ltd. for their important contributions to the development of the body pillow.

Nishikawa Co., Ltd. and Japan Society for the Promotion of Science (Grantin-Aid for Scientific Research (B) 20H04120)

\section{Conflicts of Interest}

This study was financially supported by Nishikawa Co., Ltd. MS has occasionally given lectures on sleep physiology and hygiene at educational seminars supported by Nishikawa Co., Ltd.

\section{Authors' Contribution}

Conceptualization: Park IS, Kokubo T, Kawana F, Yanagisawa M, Satoh M. Data curation: Park IS, Suzuki C, Yajima K. Formal analysis: Suzuki Y. Funding acquisition: Kokubo T, Yanagisawa M. Project administration: Fukusumi S. Writing_original draft: Park IS, Tokuyama K. Writing-review \& editing: Satoh M.

\section{REFERENCES}

1. Young T, Palta M, Dempsey J, Skatrud J, Weber S, Badr S. The occurrence of sleep-disordered breathing among middle-aged adults. $N$ Engl J Med 1993;328:1230-5.

2. Quan SF, Gersh BJ; National Center on Sleep Disorders Research; National Heart, Lung, and Blood Institute. Cardiovascular consequences of sleep-disordered breathing: past, present and future: report of a workshop from the National Center on Sleep Disorders Research and the National Heart, Lung, and Blood Institute. Circulation 2004;109:951-7.

3. Martínez-García MA, Soler-Cataluña JJ, Ejarque-Martínez L, Soriano Y, Román-Sánchez P, Illa FB, et al. Continuous positive airway pressure treatment reduces mortality in patients with ischemic stroke and obstructive sleep apnea: a 5-year follow-up study. Am J Respir Crit Care Med 2009;180:36-41.

4. Qaseem A, Holty JE, Owens DK, Dallas P, Starkey M, Shekelle P; Clinical Guidelines Committee of the American College of Physicians. Management of obstructive sleep apnea in adults: a clinical practice guideline from the American College of Physicians. Ann Intern Med 2013;159: 471-83.

5. Kushida CA, Nichols DA, Holmes TH, Quan SF, Walsh JK, Gottlieb DJ, et al. Effects of continuous positive airway pressure on neurocognitive function in obstructive sleep apnea patients: the Apnea Positive Pressure Long-term Efficacy Study (APPLES). Sleep 2012;35:1593-602.

6. Rajagopal KR, Bennett LL, Dillard TA, Tellis CJ, Tenholder MF. Overnight nasal CPAP improves hypersomnolence in sleep apnea. Chest 1986;90:172-6.

7. Zorick F, Merlotti L, Conway W, Fujita S, Rosenthal L, Roth T. Patient response to various treatments for sleep apnea. Sleep Res 1988;17:270.

8. Aldrich M, Eiser A, Lee M, Shipley JE. Effects of continuous positive airway pressure on phasic events of REM sleep in patients with obstructive sleep apnea. Sleep 1989;12:413-9.

9. Issa FG, Sullivan CE. The immediate effects of nasal continuous positive airway pressure treatment on sleep pattern in patients with obstructive sleep apnea syndrome. Electroencephalogr Clin Neurophysiol 
1986;63:10-7.

10. Weaver TE, Grunstein RR. Adherence to continuous positive airway pressure therapy: the challenge to effective treatment. Proc Am Thorac Soc 2008;5:173-8.

11. Aldrich MS. Sleep Medicine. New York: Oxford University Press, 1999.

12. Cartwright RD. Effect of sleep position on sleep apnea severity. Sleep 1984;7:110-4.

13. Richard W, Kox D, den Herder C, Laman M, van Tinteren H, de Vries $\mathrm{N}$. The role of sleep position in obstructive sleep apnea syndrome. Eur Arch Otorhinolaryngol 2006;263:946-50.

14. Oksenberg A. Positional and non-positional obstructive sleep apnea patients. Sleep Med 2005;6:377-8.

15. Cartwright RD. Effect of sleep position on sleep apnea-a review of the literature. Sleep Breath 1984;17(1):39-49.

16. Cartwright RD, Diaz F, Lloyd S. The effects of sleep posture and sleep stage on apnea frequency. Sleep 1991;14:351-3.

17. van Maanen JP, Richard W, Van Kesteren ER, Ravesloot MJ, Laman DM, Hilgevoord AA, et al. Evaluation of a new simple treatment for positional sleep apnoea patients. J Sleep Res 2012;21:322-9.

18. Bignold JJ, Deans-Costi G, Goldsworthy MR, Robertson CA, McEvoy $\mathrm{D}$, Catcheside PG, et al. Poor long-term patient compliance with the tennis ball technique for treating positional obstructive sleep apnea. $J$ Clin Sleep Med 2009;5:428-30.

19. Choi JH, Park YH, Hong JH, Kim SJ, Park DS, Miyazaki S, et al. Efficacy study of a vest-type device for positional therapy in position dependent snorers. Sleep Biol Rhythms 2009;7:181-7.

20. Permut I, Diaz-Abad M, Chatila W, Crocetti J, Gaughan JP, D'Alonzo GE, et al. Comparison of positional therapy to CPAP in patients with positional obstructive sleep apnea. J Clin Sleep Med 2010;6:238-43.

21. Kayaba M, Ogata H, Park I, Ishihara A, Kawana F, Kokubo T, et al. Improvement of slow wave sleep continuity by mattress with better body pressure dispersal. Sleep Med Res 2019;10:75-82.

22. Horne JA, Ostberg O. A self-assessment questionnaire to determine morningness-eveningness in human circadian rhythms. Int J Chronobiol 1976;4:97-110.

23. American Academy of Sleep Medicine. The AASM manual for the scoring of sleep and associated events: summary of updates in version 2.3 [cited 2010 Apr 1]. Available from: http://www.aasmnet.org/resources/pdf/scoringmanualupdates_April_2016.pdf.

24. Yamamoto Y, Tanaka H, Takase M, Yamazaki K, Azumi K, Shirakawa S. Standardization of revised version of OSA sleep inventory for mid- dle age and aged. Brain Sci Mental Disord 1999;10:401-409.

25. Tokuyama K, Ogata H, Katayose Y, Satoh M. Algorithm for transient response of whole body indirect calorimeter: deconvolution with a regularization parameter. J Appl Physiol 2009;106:640-50.

26. Park I, Ochiai R, Ogata H, Kayaba M, Hari S, Hibi M, et al. Effects of subacute ingestion of chlorogenic acids on sleep architecture and energy metabolism through activity of the autonomic nervous system: a randomised, placebo-controlled, double-blinded cross-over trial. $\mathrm{Br} \mathrm{J}$ Nutr 2017;117:979-84.

27. Ferrannini E. The theoretical bases of indirect calorimetry: a review. Metabolism 1988;37:287-301.

28. Oksenberg A, Silverberg D, Offenbach D, Arons E. Positional therapy for obstructive sleep apnea patients: a 6-month follow-up study. $\mathrm{La}$ ryngoscope 2006;116:1995-2000.

29. Bidarian-Moniri A, Nilsson M, Rasmusson L, Attia J, Ejnell H. The effect of the prone sleeping position on obstructive sleep apnoea. Acta Otolaryngol 2015;135:79-84.

30. Bidarian-Moniri A, Nilsson M, Attia J, Ejnell H. Mattress and pillow for prone positioning for treatment of obstructive sleep apnoea. Acta Otolaryngol 2015;135:271-6.

31. Joosten SA, Landry SA, Sands SA, Terrill PI, Mann D, Andara C, et al. Dynamic loop gain increases upon adopting the supine body position during sleep in patients with obstructive sleep apnoea. Respirology 2017; 22:1662-9.

32. Katz S, Arish N, Rokach A, Zaltzman Y, Marcus EL. The effect of body position on pulmonary function: a systematic review. BMC Pulm Med 2018;18:159.

33. Hewitt N, Bucknall T, Faraone NM. Lateral positioning for critically ill adult patients. Cochrane Database Syst Rev 2016;2016:CD007205.

34. Tuladhar R, Harding R, Cranage SM, Adamson TM, Horne RS. Effects of sleep position, sleep state and age on heart rate responses following provoked arousal in term infants. Early Hum Dev 2003;71:157-69.

35. Nelson EA, Taylor BJ, Weatherall IL. Sleeping position and infant bedding may predispose to hyperthermia and the sudden infant death syndrome. Lancet 1989;1:199-201.

36. Lorrain D, De Koninck J, Dionne H, Goupil G. Sleep positions and postural shifts in elderly persons. Percept Mot Skills 1986;63(2 Pt 1): 352-4.

37. De Koninck J, Lorrain D, Gagnon P. Sleep positions and position shifts in five age groups: an ontogenetic picture. Sleep 1992;15:143-9. 